\title{
10q22.3q23.3 microdeletion syndrome
}

INSERM

\section{Source}

INSERM. (1999). Orphanet: an online rare disease and orphan drug data base.

10q22.3q23.3 microdeletion syndrome. ORPHA:276413

$10 q 22.3 q 23.3$ microdeletion syndrome is a rare partial autosomal monosomy

characterized by a mild facial dysmorphism variably including macrocephaly, broad forehead, hypertelorism or hypotelorism, deep-set eyes, upslanting or downslanting palpebral fissures, low-set ears, flat nasal bridge, smooth philtrum, thin upper lip), cleft palate, cerebellar and cardiac malformations, psychomotor development delay, and behavioral abnormalities (attention deficit hyperactivity disorder, autism). Other rare features may include congenital breast aplasia, arachnodactyly, joint hyperlaxity, club feet, feeding difficulties, failure to thrive. 